\title{
Miniature FBG-based fluidic flowmeter to measure hot oil and water
}

\author{
Zhengyong Liu ${ }^{* a}$, Lin Htein ${ }^{\mathrm{a}}$, Lun-Kai Cheng ${ }^{\mathrm{b}}$, Quincy Martina ${ }^{\mathrm{b}}$, Rob Jansen ${ }^{\mathrm{b}}$, and Hwa-Yaw Tam ${ }^{\mathrm{a}}$ \\ ${ }^{a}$ Photonics Research Center, Department of Electrical Engineering, The Hong Kong Polytechnic \\ University, Hung Hom, Kowloon, Hong Kong, China; ${ }^{b}$ TNO, Stieltjesweg 1, 2628 CK Delft, The \\ Netherlands
}

\begin{abstract}
In this paper, we present a miniature fluidic flowmeter based on a packaged FBG and laser-heated fibers. The flow rates of water and hydraulic oil were measured by utilizing the proposed flowmeter. The measured results exhibited good sensitivity of $0.339 \mathrm{~nm} /(\mathrm{m} / \mathrm{s})$ for water and $0.578 \mathrm{~nm} /(\mathrm{m} / \mathrm{s})$ for oil flow. Experimental results showed that the sensitivity of the fluidic flow sensor is depending on the heat capacity of the fluids, where the fluid with higher heat capacity has higher sensitivity and lower detection limit at the same measurement condition. The real-time flow rates measured by the proposed sensor and a commercial flowmeter installed in the test rig were also compared, demonstrating good agreement with correlation coefficient of 0.9974 .
\end{abstract}

Keywords: Fiber Bragg gratings (FBGs), flow rate sensor, $\mathrm{Co}^{2+}$-doped fibers

\section{INTRODUCTION}

Fiber Bragg grating (FBGs) have been developed into various types of fiber-optic sensors to measure physical or biomedical parameters, such as strain, temperature, pressure, vibration, refractive index, molecular concentration, etc ${ }^{1}$. Because of the advantages of fast response, accurate detection, immunity to electromagnetic interference (EMI), and long-distance measurement, FBG sensors possess unique characteristics that conventional electrical-based sensors don't have. It has been reported that the temperature of FBG can be heated up by launching high power laser light into the fiber and then absorbed by the metal film (e.g. silver) coated on the surface of the grating ${ }^{2,3,4,5}$. This type of device was demonstrated to measure the wind/gas flow as a hot-wire anemometry ${ }^{6}$. However, to heat the FBG, additional fibers or structures (such as multimode fiber ${ }^{3}, \mathrm{LPG}^{4}$, no-core fiber ${ }^{5}$ ) are required so as to leak out the laser light from the core. Recently, we demonstrated another heating scheme by using $\mathrm{Co}^{2+}$-doped fibers ${ }^{7,8}$, on which FBG can be inscribed and heated by the guided laser light directly. Good performance of measuring wind speed was obtained by detecting the heat loss on the self-heated FBG. Because the gas or air has very low heat capacity, it is very easy to heat the FBG to high temperature (e.g. hundreds of Celsius degrees). However, more laser power is needed to heat the FBG if placed into liquids, which generally have much higher heat capacity and viscosity as well, similar to the electrically-heated flow sensor ${ }^{9}$. In order to measure the fluidic flow rates, special design of heating configuration is needed, which is also driven by the requirements of precise measurement of the flow rates of hot oil, hot water and even the mixed phase of both in oil industry.

In general, hot-wire/films based on electrical heating are used to measure liquids flow rate, where the resolution varies from $0.002 \mathrm{~m} / \mathrm{s}$ to $0.5 \mathrm{~m} / \mathrm{s}$, depending on the design and structures of the flow sensors ${ }^{10}$. Most of the micromachined flow sensors are aiming for the measurement of microfluidics. It is worthy to mention that in addition to thermal methods, FBGs are also employed to measure fluids' flow rate by detecting the force vertically applied on the grating or measuring the time delay of fluidic vortex signal ${ }^{11,12,13}$. In this paper, we present a novel thermal flow sensors integrated with a short FBG heated up by one or several $\mathrm{Co}^{2+}$-doped multimode fibers. The comparison of flow measurements is conducted for the single phase of water and hydraulic oil. Experimental results demonstrate that larger temperature difference between the flow sensor and oil can be obtained than water due to the higher heat capacity of oil. Particularly, the measured resolution of oil flow is $0.002 \mathrm{~m} / \mathrm{s}$, which is lower than that in water, i.e. $0.005 \mathrm{~m} / \mathrm{s}$. Because of the fast response and accurate discrimination of flow rate, the proposed miniature sensor is a potential candidate to measure fluidic flow in oil industry. Since FBG is utilized, it is also easy to implement multiplexing of a large number of the proposed flow sensors along the measurement path, which can be measured over very long distance.

* zhengyong.liu@connect.polyu.hk; phone: +852 27664678

25th International Conference on Optical Fiber Sensors, edited by Youngjoo Chung, Wei Jin,

Byoungho Lee, John Canning, Kentaro Nakamura, Libo Yuan, Proc. of SPIE Vol. 10323,

$1032336 \cdot$ (C) 2017 SPIE · CCC code: 0277-786X/17/\$18 · doi: 10.1117/12.2264880 


\section{CHARACTERISTICS OF THE FLUIDIC FLOWMETER}

Fig. 1 shows the schematic figure of the proposed flow sensor, which consists of a short FBG written on single mode fiber surrounded by four identical $\mathrm{Co}^{2+}$-doped multimode fibers, which have core diameter of $50 \mu \mathrm{m}$. The length of FBG is only $3 \mathrm{~mm}$ to avoid chirp effect after being heated, and the $\mathrm{Co}^{2+}$-doped fibers are designed to have a length of $5 \mathrm{~mm}$ so as to surround the entire FBG. All the assembly is protected by a stainless steel tube having inner and outer diameter of $380 \mu \mathrm{m}$ and $500 \mu \mathrm{m}$, respectively, in which the position of FBG and $\mathrm{Co}^{2+}$-doped fibers are precisely controlled. Note that due to the large core size of $\mathrm{Co}^{2+}$-doped fibers used, the temperature of the $\mathrm{FBG}$ can be easily heated up to hundreds of Celsius degrees (e.g. $\sim 900{ }^{\circ} \mathrm{C}$ in air) by pumping the laser light with high optical power into multimode fibers. Even inside the fluids, temperature difference of about $200^{\circ} \mathrm{C}$ can be obtained, this is highly depending on the heat capacity of the fluids.

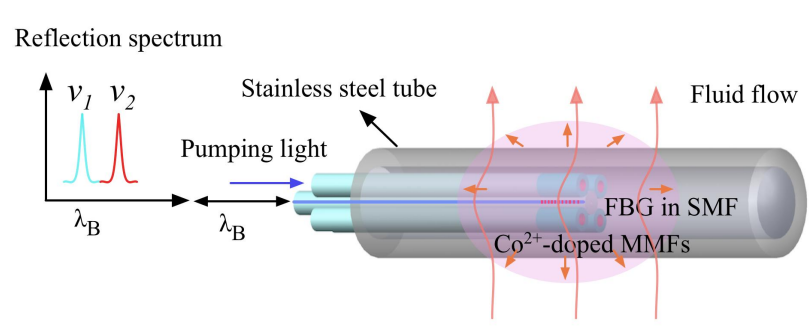

(a)

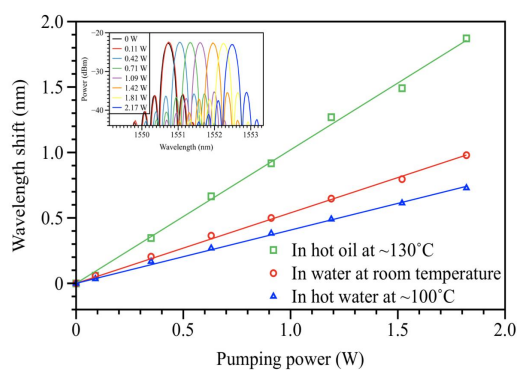

(b)

Fig. 1 (a) Schematic figure of the fluidic flow sensor consisting of a short FBG surrounded by four identical $\mathrm{Co}^{2+}$-doped multimode fibers with a length of $5 \mathrm{~mm}$, and (b) wavelength shifts as a function of pumping power when the flow sensor is placed inside the water at room temperature and $\sim 100^{\circ} \mathrm{C}$, and hot oil at $\sim 130{ }^{\circ} \mathrm{C}$, the inset shows the spectrum shift of FBG in the hot oil

To characterize the temperature response with respect to pumping power inside different fluids, the flow sensor was placed inside water at room temperature, water at $\sim 100^{\circ} \mathrm{C}$ and hydraulic oil at $\sim 130^{\circ} \mathrm{C}$, separately. Then the laser power was gradually increased to monitor the wavelength shift of FBG. The results are plotted in Fig. 1(b). It can be observed that even though at different fluidic conditions, the temperature increase follows a reasonable linear relationship with respect to the pumping power, which is similar to the heating process of hot fibers ${ }^{2,8,14}$. On the same amount of pumping power, the temperature difference between the flow sensor and oil is larger than water because the heat capacity of the former fluid is $\sim 1.88 \mathrm{Jg}^{-1} \mathrm{~K}^{-1}$, which is about 2 times lower than water, i.e. $4.18 \mathrm{Jg}^{-1} \cdot \mathrm{K}^{-1}$. The small difference of cold and hot water is probably due to slight change of heat capacity between the two phases of water. Taking the coefficient of wavelength shift of FBG versus the temperature into account, i.e. $10.7 \mathrm{pm} /{ }^{\circ} \mathrm{C}$ in experiment, about $200{ }^{\circ} \mathrm{C}$ temperature difference can be easily obtained inside the oil by pumping less than $2 \mathrm{~W}$ laser light. Note that even higher temperature difference can be achieved for oil and water phase, depending on the measurement requirements. The large temperature increase achieved in hot fluids makes it possible to measure the flow rates of hot oil and water in harsh environment.

When the heated flow sensor is subjected to dynamic fluidic flow, heat is carried away by the fluids, which therefore reduce the temperature of the sensor head due to the heat convection. The FBG in the flow sensor functions as the temperature sensor. According to the principle of hot-wire anemometry and temperature dependence of FBG, the relationship between the Bragg wavelength and the flow velocity can be expressed by

$$
\lambda=\lambda_{0}+2 n_{\text {eff }} \Lambda(\alpha+\beta) \frac{H_{\text {loss }}}{A+B \cdot \sqrt{v}},
$$

where $\alpha$ is the coefficient of thermal expansion of silica, $\beta$ is the thermal-optic coefficient, $\Lambda$ is the pitch of FBG, and $n_{\text {eff }}$ is the effective index of the guided mode of the fiber; $A, B$ are the empirical constants for the heat transfer of specific fluid, and $H_{\text {loss }}$ represents the heat loss at a given flow speed of $v$.

\section{EXPERIMENTAL RESULTS OF FLOW MEASUREMENT}

To demonstrate the performance of the proposed sensor to monitor the fluidic flow, the flow sensor was installed in a Plexiglas tube of a test rig at TNO, The Netherlands, as shown in Fig. 2(a). The Plexiglas tube has an inner diameter of 
$25 \mathrm{~mm}$, therefore an oil mass flow rate of $100 \mathrm{~kg} / \mathrm{h}$ corresponds to $0.064 \mathrm{~m} / \mathrm{s}$ in velocity. Fig. 2(b) plots the wavelength shift of measuring oil and water flow by increasing the flowing rates, respectively, where the pumping laser power was kept at $1.43 \mathrm{~W}$. Due to the difference heat capacity of oil and water, the initial temperature increased at zero flow rate is different. The response of oil flow exhibits relatively higher sensitivity. For each step change of the flowing rate, the temperature of sensor head (Bragg wavelength) can get stabilized when the heat convection is in equilibrium. It can be seen that when the fluid started flowing pass the sensor surface, the temperature of the sensor decreased rapidly because of the heat convection. This also implies that the sensor is very sensitive to slow flowing rates. The measured data was fitted well via the Eq. (1). By calculating the first derivative of the fitted curve, the sensitivity can be estimated to be, for instance, $0.409 \mathrm{~nm} /(\mathrm{m} / \mathrm{s})$ for oil flow and $0.22 \mathrm{~nm} /(\mathrm{m} / \mathrm{s})$ for water flow at the rate of $0.2 \mathrm{~m} / \mathrm{s}$. The sensitivity varies at different rates owing to the non-linear response. At this particular condition of flowing rate and pumping power, the detection limit of measuring water and oil flow is $0.005 \mathrm{~m} / \mathrm{s}$ and $0.002 \mathrm{~m} / \mathrm{s}$, respectively, considering the resolution of interrogator used is 1 pm. Fig. 2 (b) also shows that larger initial temperature difference gives higher measurement sensitivity.

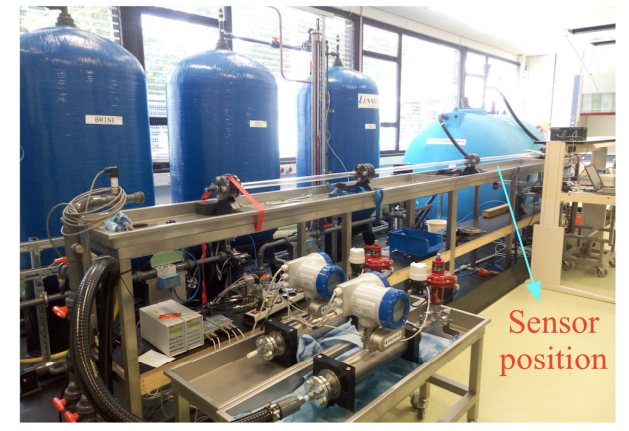

(a)

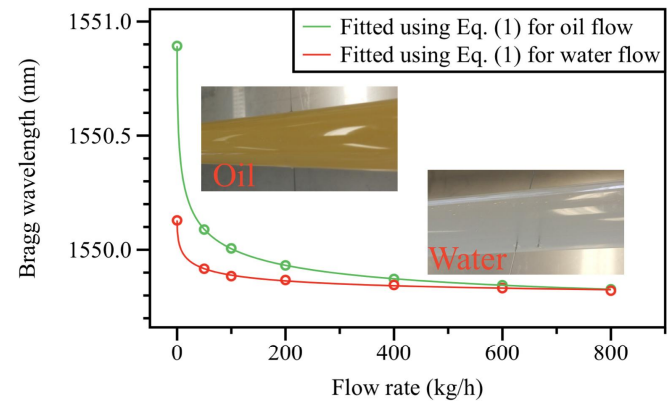

(b)

Fig. 2 (a) Photo of the test rig of oil and water flow measurement, and (b) the measured wavelength curves as functions of flow rates for oil and water at the same pumping power of $1.43 \mathrm{~W}$, where the inset show the photos of oil and water inside the tube, respectively.

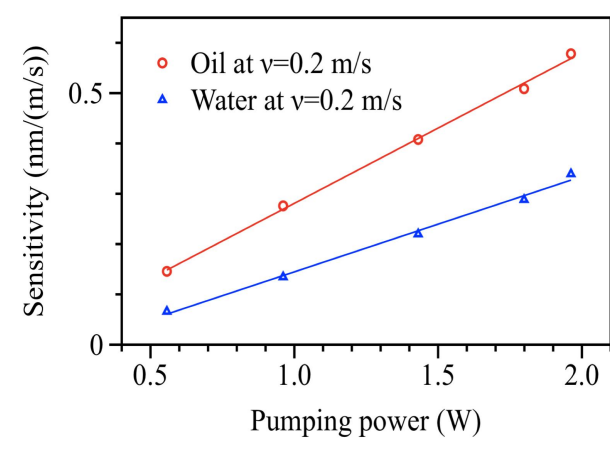

(a)

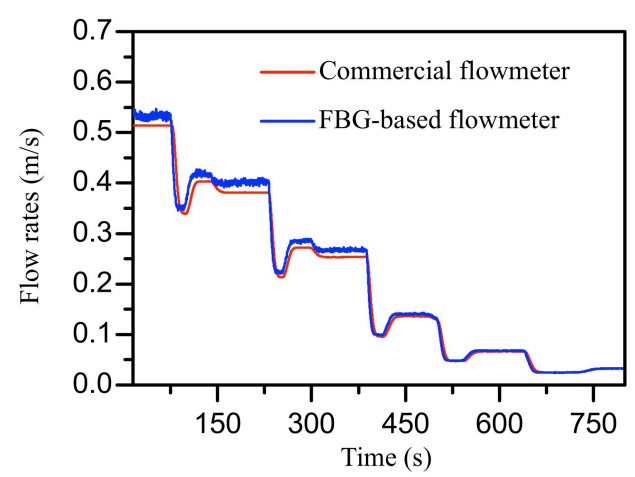

(b)

Fig. 3 (a) Comparison of sensitivity of measuring oil and water flow at the same rate of $0.2 \mathrm{~m} / \mathrm{s}$ as a function of pumping power, (b) comparison curves of flow rates measured using the proposed FBG-based sensor and a commercial flowmeter installed inside the test rig

Therefore the sensitivity can be improved further by increasing the pumping laser power, as illustrated in Fig. 3(a), where the sensitivity at the rate of $0.2 \mathrm{~m} / \mathrm{s}$ for oil and water flow is plotted with respect to various pumping powers. Higher optical power means more light energy can be converted to heat at the sensing head, and thus leading to larger initial temperature difference, which is applicable for the fluids with higher heat capacity, i.e. water. Compared with water flow, it is relatively easy to get better sensitivity and resolution to measure oil flow by launching larger laser power. After the calibration of the flow sensor, the real time monitoring of oil flow was conducted. The results are plotted in Fig. 3(b), where the flow rates measured by a commercial flowmeter installed in the test rig is also plotted for comparison. It can be seen that the heat-FBG flow sensor can discriminate very slight change in flow-rate, even the overshoot dip can 
be distinguished before the flow rate gets stable. The correlation of the flow rates measured by those two flowmeters is calculated to be 0.9974 , indicating the good accuracy of the proposed sensor.

\section{CONCLUSION}

In conclusion, a novel fluidic flowmeter based on a compact integration of heated-FBG and $\mathrm{Co}^{2+}$-doped fibers is demonstrated. The experimental results of measuring hot water and hot oil flow rates are shown. The sensor shows different performance for the fluids with different heat capacities, which determines the initial heating temperature of the sensor. Higher pumping laser power results in larger initial temperature difference between the sensor and fluids, and eventually influences the sensitivity. The heated-FBG sensor has comparable to conventional electrical sensor and a correlation coefficient of 0.9974 for the measured results using both types of sensors is obtained experimentally. As single phase of water and oil is characterized individually here, it is also important to analyze the mixed phase of the fluids with high temperature as a view of practical use in oil industry, which is under investigation. The advantages of FBGs as well as the promising results make the proposed sensor a good candidate in flow monitoring of oil and gas industry.

\section{ACKNOWLEDGEMENT}

The authors would like to acknowledge the support of Research Grant Council General Research Fund, project number PolyU5246/13E.

\section{REFERENCES}

[1] A. D. Kersey et al., "Fiber grating sensors," Journal Article, IEEE J. Light. Technol. 15(8), 1442-1463 (1997).

[2] K. P. Chen et al., "Self-heated fiber Bragg grating sensors," Appl. Phys. Lett. 86(14), 1-3 (2005).

[3] L. J. Cashdollar and K. P. Chen, "Fiber Bragg grating flow sensors powered by in-fiber light," IEEE Sens. J. 5(6), 1327-1331 (2005).

[4] P. Caldas et al., "Fiber optic hot-wire flowmeter based on a metallic coated hybrid long period grating/fiber Bragg grating structure," Appl. Opt. 50(17), 2738-2743 (2011).

[5] X. Wang et al., "Hot-wire anemometer based on silver-coated fiber Bragg grating assisted by no-core fiber," IEEE Photonics Technol. Lett. 25(24), 2458-2461 (2013).

[6] H. H. Bruun, Hot-Wire Anemometry: Principles and Signal Analysis, Oxford University Press (1995).

[7] S. Gao et al., "All-optical fiber anemometer based on laser heated fiber Bragg gratings," Journal Article, Opt. Express 19(11), 10124-10130, OSA (2011).

[8] Z. Liu et al., "Integrated microfluidic flowmeter based on a micro-FBG inscribed in Co2+-doped optical fiber," Opt. Lett. 39(20), 5877-5880 (2014).

[9] R. Ahrens and K. Schlote-Holubek, "A micro flow sensor from a polymer for gases and liquids," J. Micromechanics Microengineering 19(7), 74006 (2009).

[10] J. T. W. Kuo, L. Yu, and E. Meng, "Micromachined Thermal Flow Sensors-A Review," Micromachines 3(4), 550-573 (2012).

[11] S. Takashima, H. Asanuma, and H. Niitsuma, "A water flowmeter using dual fiber Bragg grating sensors and cross-correlation technique," Sensors Actuators A Phys. 116(1), 66-74 (2004).

[12]L. K. Cheng et al., "Development of a FBG vortex flow sensor for high-temperature applications," in Proc. SPIE 7753, 21st Int. Conf. Opt. Fiber Sensors, p. 77536V (2011).

[13] V. Baroncini et al., "Single- and Two-Phase Flow Characterization Using Optical Fiber Bragg Gratings," Sensors 2015(15), 6549-6559 (2015).

[14] Y. Li et al., "Microfluidic flowmeter based on micro 'hot-wire' sandwiched Fabry-Perot interferometer," Opt. Express 23(7), 9483 (2015). 